\title{
Supporting information: Deep learning driven GC-MS library search and its application for metabolomics
}

\author{
Dmitriy D. Matyushin*, Anastasia Yu. Sholokhova, Aleksey K. Buryak
}

A.N. Frumkin Institute of Physical Chemistry and Electrochemistry, Russian Academy of Sciences E-mail: dm.matiushin@mail.ru

\section{Table of contents}

Supporting information, file 1 (this file):

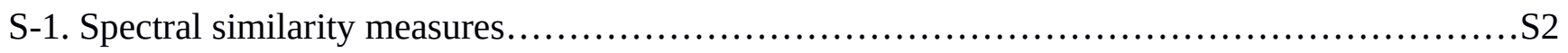

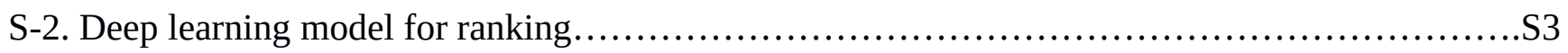

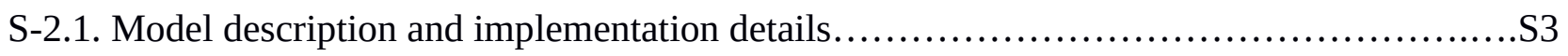

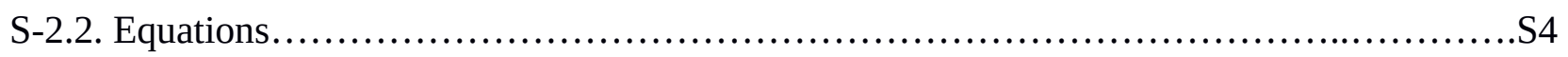

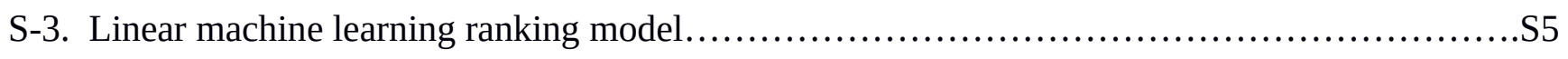

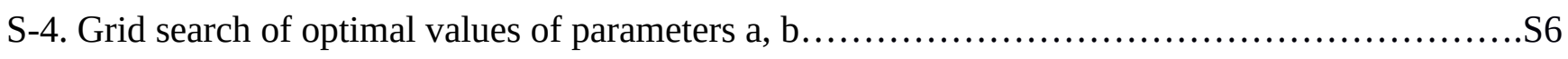

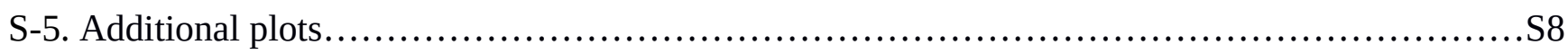

Supporting information, file 2 (xlsx spreadsheet): Information about data sets (.xlsx)

Supporting information, file 3 (xlsx spreadsheet): Complete results of search accuracy evaluations (rank-1 - rank-100) for all data sets and search algorithms (.xlsx)

Pre-trained model parameters, source code and corresponding instructions are available online: https://www.doi.org/10.6084/m9.figshare.12685790 


\section{S-1. Spectral similarity measures}

Let $\left(q_{1}, q_{2} \ldots q_{M}\right)$ and $\left(r_{1}, r_{2} \ldots r_{M}\right)$ be query and reference mass spectra respectively; $1 \ldots M-m / z$ range; $q_{n}$ and $r_{n}$ - intensities corresponding to $\mathrm{m} / \mathrm{z}=n ; N_{Q}, N_{Q R}$ - number of peaks in a query spectrum and in both spectra simultaneously; $n_{i}-m / z$ of $i$-th peak that is present in both spectra simultaneously.

Euclidean distance spectral similarity measure $S_{e}$ and Manhattan (absolute value distance) spectral similarity measure $S_{m}$ :

$S_{e}(a, b)=\left(1+\frac{\sum_{n=1}^{M}\left(n^{a} q_{n}^{b}-n^{a} r_{n}^{b}\right)^{2}}{\sum_{n=1}^{M}\left(n^{a} q_{n}^{b}\right)^{2}}\right)^{-1} ; S_{m}(a, b)=\left(1+\frac{\left.\sum_{n=1}^{M} \mid n^{a} q_{n}^{b}-n^{a} r_{n}^{b}\right)}{\sum_{n=1}^{M} n^{a} q_{n}^{b}}\right)^{-1}$

As input for a linear ranking model, we also use recall, precision, weighted recall, weighted precision. These values also characterize spectral similarity. However, direct usage of these values as spectral similarity for a library search gives very poor results.

recall $=\frac{N_{Q R}}{N_{R}} ;$ precision $=\frac{N_{Q R}}{N_{Q}} ;$ weighted recall $=\frac{\sum_{i=1}^{i=N_{Q R}} n_{i}^{a} r_{n_{i}}^{b}}{\sum_{n=1}^{n=M} n^{a} r_{n}^{b}} ;$ weighted precision $=\frac{\sum_{i=1}^{i=N_{Q R}} n_{i}^{a} q_{n_{i}}^{b}}{\sum_{n=1}^{n=M} n^{a} q_{n}^{b}}$

In all these equations, $a, b$ - adjustable constants. We use $a=1.0, b=0.5$, unless otherwise indicated.

W+DFT.R spectral similarity measure was also initially used for comparison:

$\left.S_{W+D F T . R}=\mid \frac{N_{Q} \sum_{n=1}^{M} n^{3} q_{n}^{0.5} n^{3} r_{n}^{0.5}}{\sqrt{\sum_{n=1}^{M}\left(n^{3} q_{n}^{0.5}\right)^{2} \sum_{n=1}^{M}\left(n^{3} r_{n}^{0.5}\right)^{2}}}+\frac{N_{Q R} \sum_{n=1}^{M} q_{n}^{D F T} r_{n}^{D F T}}{\sqrt{\sum_{n=1}^{M}\left(q_{n}^{D F T}\right)^{2} \sum_{n=1}^{M}\left(r_{n}^{D F T}\right)^{2}}}\right) \frac{1}{N_{Q}+N_{Q R}}$,

where $x_{n}^{D F T}=\sum_{k=1}^{M} x_{k} \cos (2 \pi k(n-1) / M)$, where $x$ means $r$ or $q$. 


\section{S-2. Deep learning model for ranking}

\section{S-2.1. Model description and implementation details}

The deep convolutional neural network for ranking has 6 1D convolutional layers, 4 subsampling layers (which are placed after the second, third, fourth, and fifth 1D convolutional layers), a reshape layer (placed after the sixth 1D convolutional layer), and 2 dense layers. All 1D convolutional layers have kernel = 6 and 300 output channels. The first 1D convolutional layer has 36 input channels. The input matrix has dimensions $750 * 36$. All subsample layers have kernel $=3$ (i.e., reduce the matrix width by 3). MAX-pooling downsampling is used. It means that for each channel, its max value in the kernel mask is used as the input value for the next layer.

The reshape layer simply rearranges the matrix-shape output from the last convolutional layer to the vector-shape input for the dense layer. The first dense layer has 1500 input nodes, 600 output nodes, and the last one (output layer) has one output node. All layers but the last one use ReLU activation function, the last one uses identity activation function. Adam algorithm was used for training with a learning rate of 0.0003; global L2-regularization $\left(l_{2}=10^{-5}\right)$ was applied. XAVIER weight initialization was used. The neural network was implemented using ComputationGraph class from Eclipse Deeplearning4j framework.

Before training, 5000 query spectra were separated from the training set and used for monitoring of training (a validation set). The network parameters for that iteration for which rank-1 search accuracy for the monitoring (validation) set is the highest are used later. When querying the monitoring (validation) set, we treat stereoisomers as different compounds, but when training the network, we consider stereoisomers as identical compounds.

Layer-wise number of weights and biases:

\begin{tabular}{|l|l|l|l|}
\hline $\mathrm{N}$ & \multicolumn{1}{|c|}{ Layer } & \multicolumn{1}{|c|}{ Weights } & \multicolumn{1}{c|}{ Biases } \\
\hline 1 & Convolutional 1 & 64800 & 300 \\
\hline 2 & Convolutional 2 & 540000 & 300 \\
\hline 3 & Convolutional 3 & 540000 & 300 \\
\hline 4 & Convolutional 4 & 540000 & 300 \\
\hline 5 & Convolutional 5 & 540000 & 300 \\
\hline 6 & Convolutional 6 & 540000 & 300 \\
\hline 7 & Dense 1 & 900000 & 600 \\
\hline 8 & Dense 2 & 600 & 1 \\
\hline Total: & 3665400 & 2401 \\
\hline
\end{tabular}

The code that initializes the neural network and describes all hyperparameters is given in “ConstantsAndHyperparameters.java” file. During training, we minimize RankNet loss function; RankNet is implemented in "RankNetLossFunction.java” file. “App.java” file contains code that 
enables reproducing all main findings of this article: importing data from databases to an internal file format, training, testing, cross-validation of the deep learning model, training and testing of the linear ranking model, evaluation of all other spectral measures. This file also contains detailed comments and can be used as an example of training and evaluation code. "Example1.java” file contains a simple training and validation example, "Example2.java” and “Example3.java” files contain simple examples of using the pre-trained model. “AppCLI.java” file contains a simple command-line application.

Pre-trained model parameters, source code and corresponding instructions are available online:

\section{https://www.doi.org/10.6084/m9.figshare.12685790}

Instructions for compiling and running a project using CPU and GPU, as well as code examples: https://ndownloader.figshare.com/files/24021353

Archive with source codes (including the source files mentioned above): https://ndownloader.figshare.com/files/24021356

Parameters of the pre-trained model: https://ndownloader.figshare.com/files/24021359

\section{S-2.2. Equations}

The input of $1 \mathrm{D}$ convolutional layer is a matrix $L^{*} M_{\text {input }}$ and the output is a matrix $(L-k+1) * M_{\text {output }}$, where $L$ - width, $k$ - kernel, $M$ - number of channels:

$$
O_{i, j}=F\left(b_{j}+\sum_{l=1}^{l=k} \sum_{m=1}^{m=M_{\text {input }}} w_{l, m, j} I_{(i+l-1), m}\right)
$$

where $I_{a, m}$ - input data (the element at $a$-th row, $m$-th column); $O_{i, j}$ - output data; $w_{l, m, j}, b_{j}$ - weights and biases (parameters optimized during learning); $F$ - activation function.

MAX-pooling subsample layer reduces the width by 3: $O_{i, j}=\max \left(I_{(3 i-2), j}, I_{(3 i-1), j}, I_{(3 i), j}\right)$

Dense layer converts a vector to a vector: $O_{j}=F\left(b_{j}+\sum_{i=1}^{i=L} w_{i, j} I_{i}\right)$

Output layer converts a vector to output relevance: Relevance $=b+\sum_{i=1}^{i=L} w_{i} X_{i}$

We use the rectified linear (ReLU) function as the activation function: $F_{\operatorname{ReLU}}(x)=\left\{\begin{array}{l}x, x>0 \\ 0, x \leqslant 0\end{array}\right.$ 
RankNet loss function is used: $C=\sum_{i=1, j=1}^{i=c, j=c}\left(\frac{1}{2}\left(1-S_{i j}\right) \sigma\left(s_{i}-s_{j}\right)+\ln \left(1+e^{-\sigma\left(s_{i}-s_{j}\right)}\right)\right)$, where $\sigma-$ adjustable constant, $c$ - number of candidates, $s_{i}$ - predicted relevance of $i$-th candidate, $S_{i j}=\left\{\begin{array}{cc}0, & a_{i}=a_{j} \\ 1, & a_{i}>a_{j} \\ -1, & a_{i}<a_{j}\end{array}\right.$, where $a_{i}$ - target relevance of $i$-th candidate: $a_{i}=1$ if a candidate is identical to a query compound or is its stereoisomer (cis/trans or optical), $a_{i}=0$ elsewhere. We use $\sigma=1.0, c=10$ in our work. Overall loss function includes L2-regularization term in addition to RankNet loss function.

\section{S-3. Linear machine learning ranking model}

The relevance of a candidate is calculated as Relevance $=b+\sum_{i=1}^{i=L} w_{i} X_{i}$

where $w_{i}, b$ - weights and a bias (parameters optimized during learning); $X_{i}$-input features; $L=42$. Input features are listed in the table:

\begin{tabular}{|l|l|}
\hline$i$ & $X_{i}$ \\
\hline $1-30$ & $\begin{array}{l}\text { Dot product and composite similarity with various values of parameters } a, b: \\
a \in(0,0.5,1,2,3) \\
b \in(0.5,0.6,1) \\
d=b\end{array}$ \\
\hline $31-38$ & Recall, precision, weighted recall, weighted precision. Two combinations of parameters: $a=0, b=1$ and $a=1, b=0.5$ \\
\hline $39-42$ & $\begin{array}{l}\text { Manhattan distance and Euclidean distance spectral similarity measures. Two combinations of parameters: } a=0, b=1 \text { and } a= \\
1, b=0.5\end{array}$ \\
\hline
\end{tabular}

The training procedure was almost exactly the same as for the deep neural network: random initialization of weights, Adam optimization algorithm with a learning rate of 0.0003 , RankNet loss function, batches that contain top-10 candidates for each query (one query spectra - one batch for the model). $10 \%$ of the data set were used as a monitoring (validation) set.

There are two differences: we use all query spectra for training and monitoring, and we do not use any regularization. We do not need to provide the absence of overlapping between training and test sets because such model (with 43 parameters) is unlikely to be overfitted with the data set that contains tens of thousands samples. 


\section{S-4. Grid search of optimal values of parameters $a, b$}

We provided the grid search of parameters $a, b$ for which maximal search accuracy can be achieved. We calculated search accuracies at various ranks (rank-1 - rank-10) for composite spectral similarity measure $(d=b)$, for the dot product and Euclidean distance spectral similarity measures. We varied parameter $a$ in the range 0.0 - 3.0 with a step of 0.25 , and we varied parameter $b$ in the range 0.3 - 0.9 with a step of 0.02 . At all ranks, for all spectral similarity measures for optimal pair of parameters: $a$ lies in the range $0.75-1.25$ and $b$ lies in the range $0.46-0.54$. In all cases, the difference between search accuracy for $a=1.0, b=0.5$ and search accuracy for optimal (for specified rank and measure) parameters $a, b$ does not exceed 0.15 percentage point and in most cases is much lower. We use values $a=1.0, b=0.5$ throughout this research, unless otherwise specified.

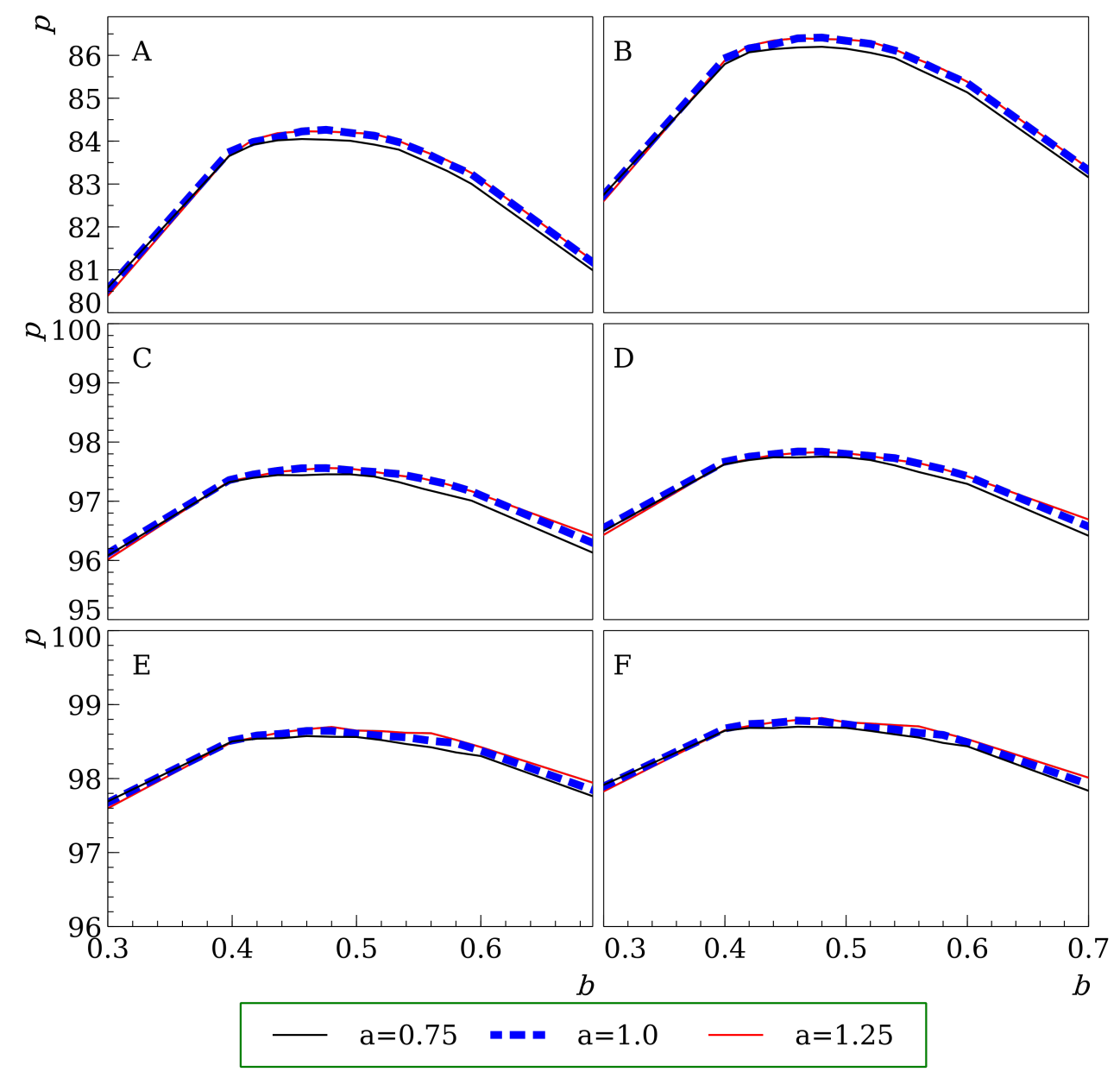

Figure S-4.1. The dependence of search accuracy $p$ on parameter $b$ with various values of parameter $a$. Composite spectral similarity was used. Search accuracy is shown at rank-1 (A, B), at rank-5 (C, D), at rank-10 (E, F). Stereoisomers were treated as different compounds (A, C, E) and as identical compounds (B, D, F). NIST 17 replicate library was used as a source of queries. 


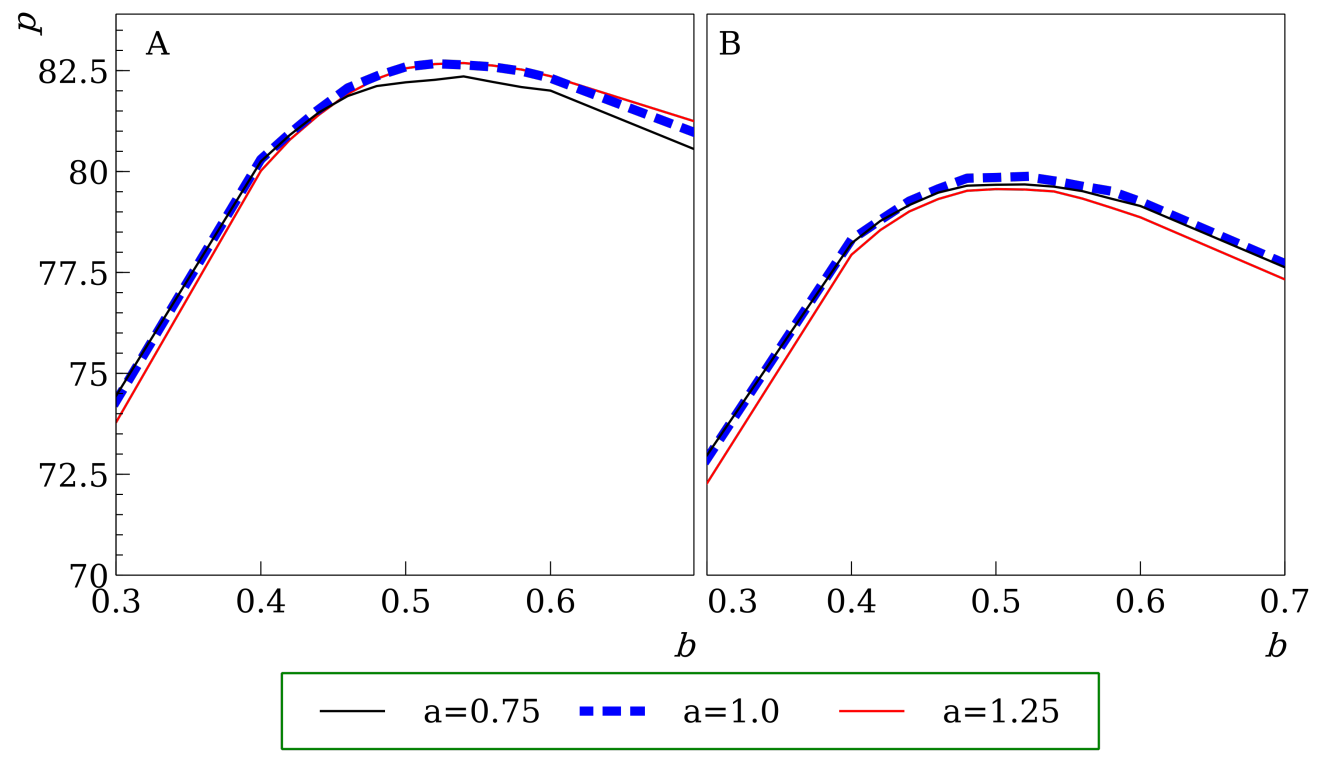

Figure S-4.2. The dependence of search accuracy $p$ on parameter $b$ with various values of parameter $a$. The dot product (A) and Euclidean distance (B) spectral similarity were used. Search accuracy is shown at rank-1. Stereoisomers were treated as different compounds. NIST 17 replicate library was used as a source of queries.
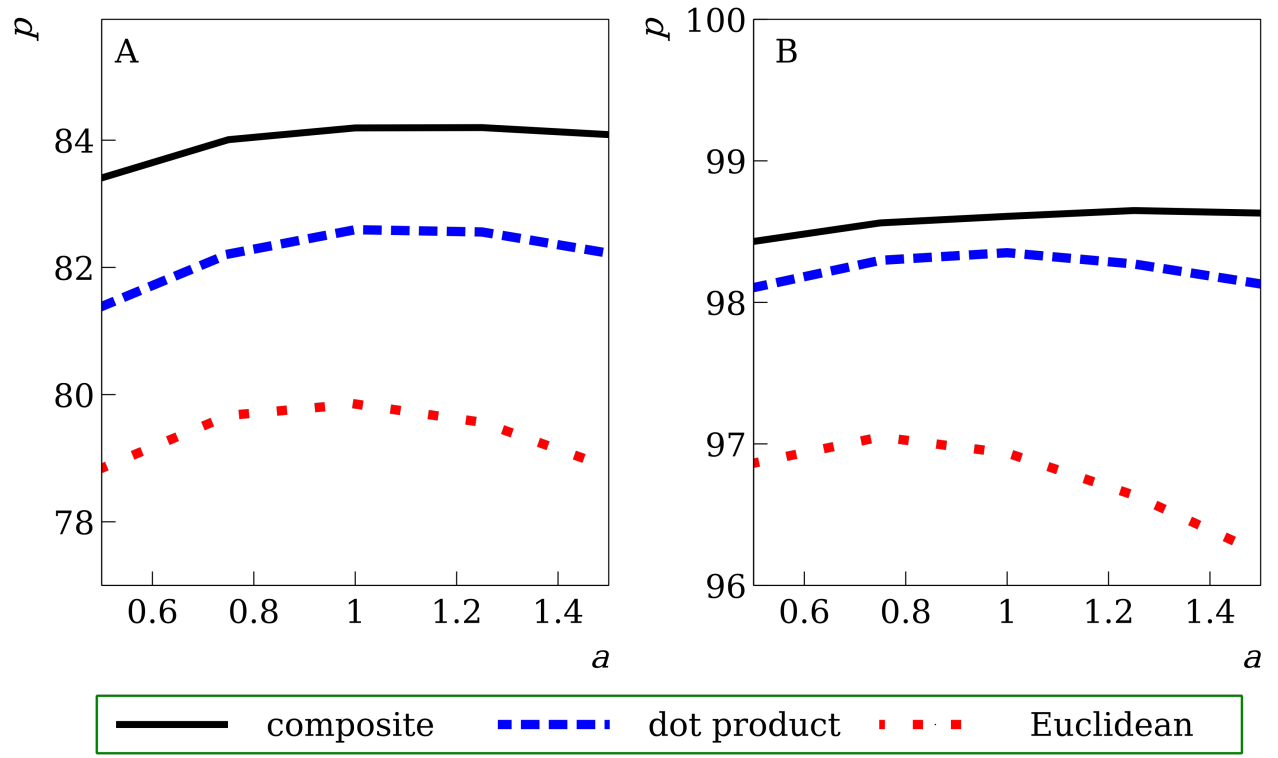

Figure S-4.3. The dependence of search accuracy $p$ on parameter $a(b=0.5)$. Various spectral similarities were used. Search accuracy is shown at rank-1 (A) and at rank-10 (B). Stereoisomers were treated as different compounds. NIST 17 replicate library was used as a source of queries. 


\section{S-5. Additional plots}
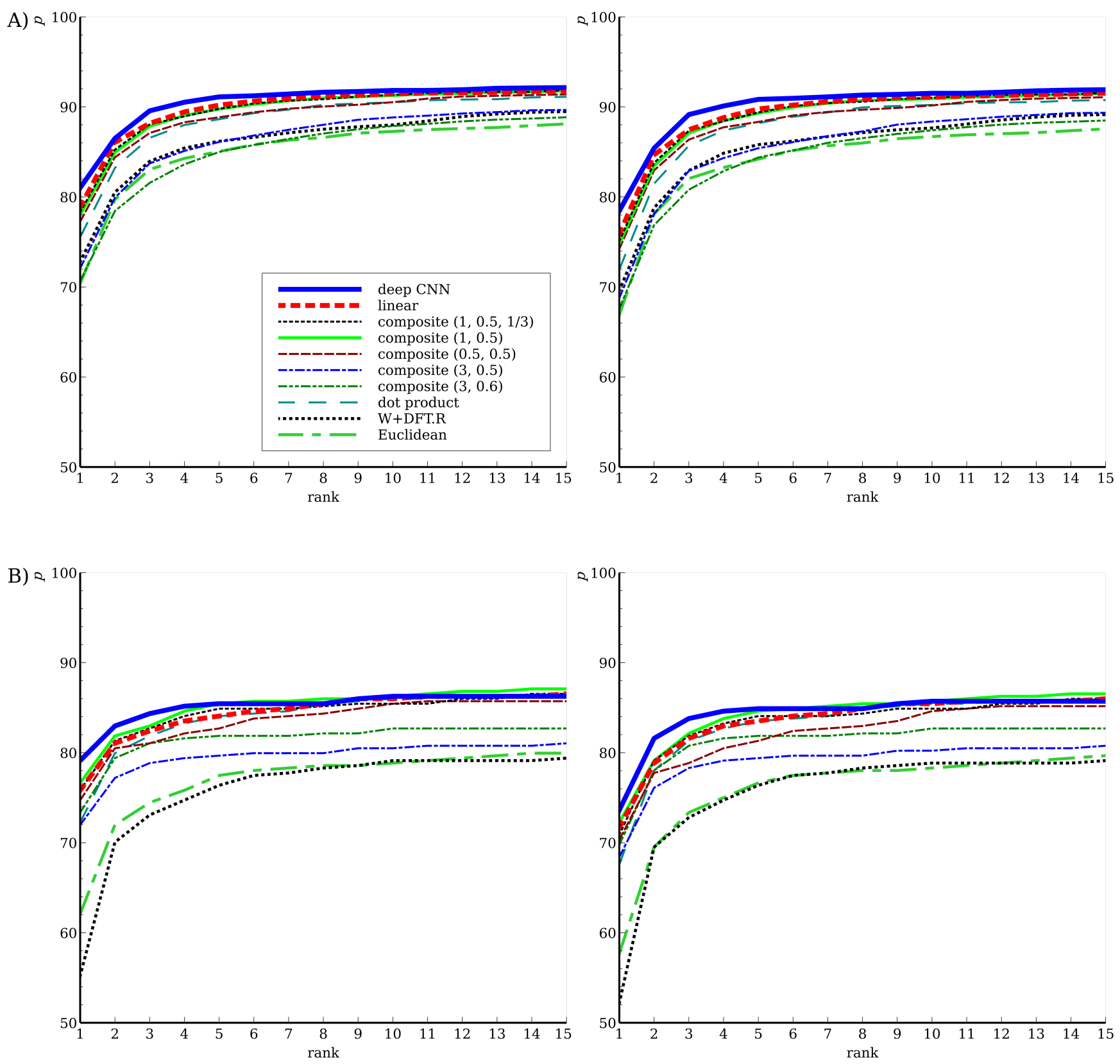

Figure S-5.1. The library search accuracy $p$ at various ranks, for various similarity measures. Query sets: HMDB (A) and FiehnLib (B); reference set: NIST 17 mass spectral library. Numbers in brackets for composite similarity measure: parameters $a, b, d$; if parameter $d$ is omitted, then $b=d$. For other similarity measures: $a=1.0, b=0.5$. The plots on the left are given for the case when stereoisomers were treated as identical compounds and the plots on the right for the case when stereoisomers were treated as different compounds. 

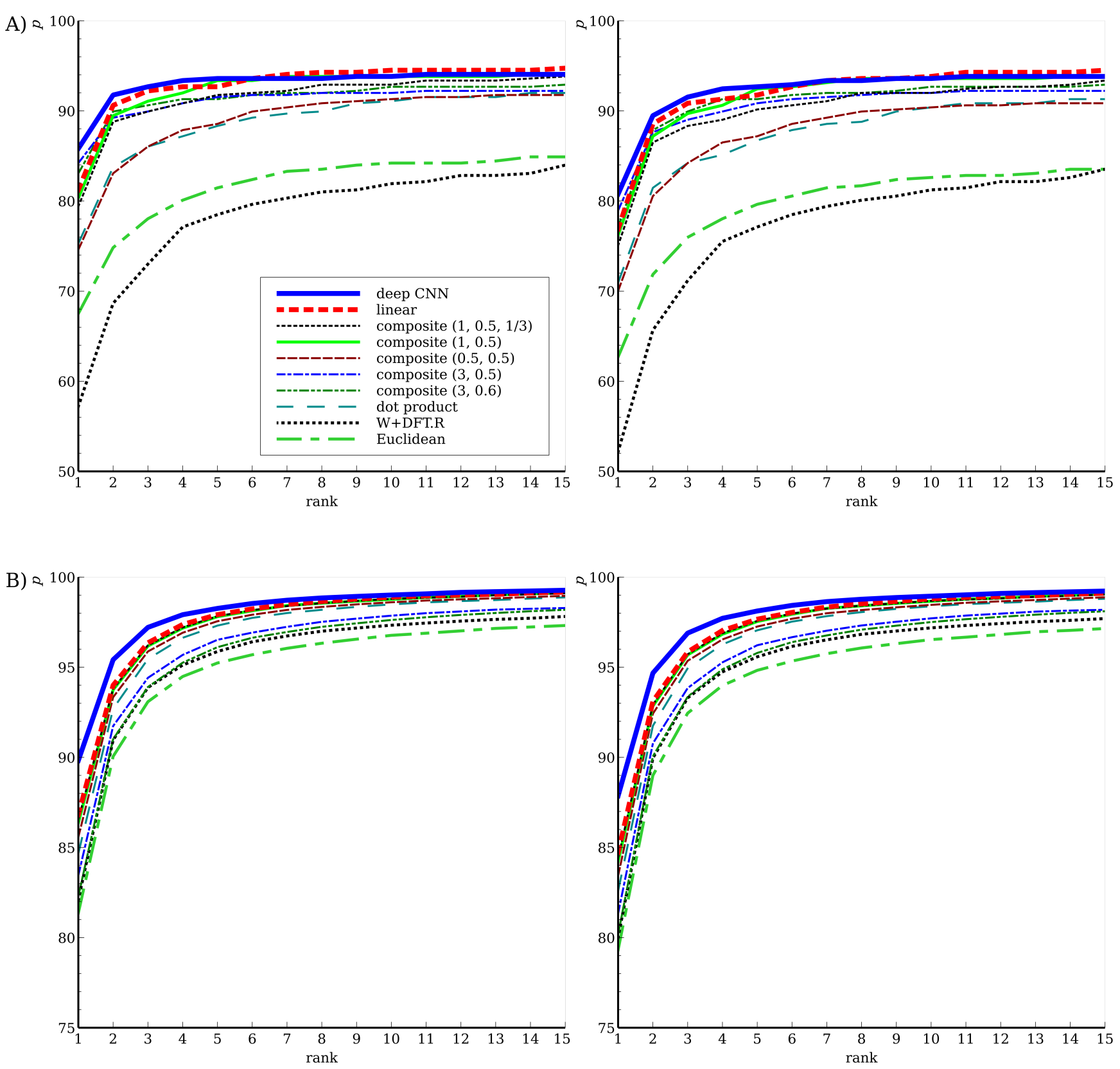

Figure S-5.2. The library search accuracy $p$ at various ranks, for various similarity measures. Query sets: GMD (A) and NIST 17 replicate library (B); reference set: NIST 17 mass spectral library. Numbers in brackets for composite similarity measure: parameters $a, b, d$; if parameter $d$ is omitted, then $b=d$. For other similarity measures: $a=1.0, b=0.5$. The plots on the left are given for the case when stereoisomers were treated as identical compounds and the plots on the right for the case when stereoisomers were treated as different compounds. Note that the different scale along the axis is used for replib and for other sets. 

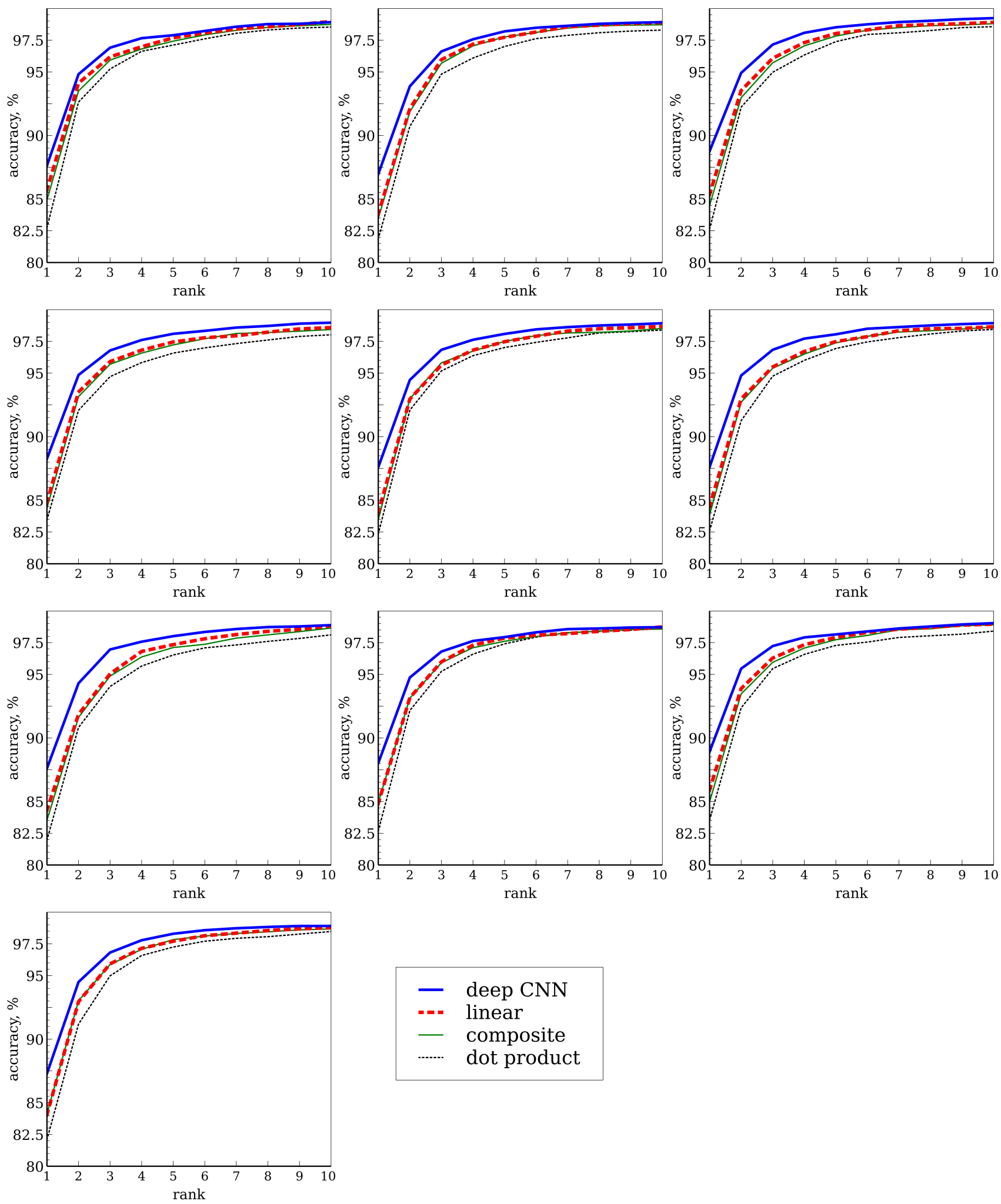

Figure S-5.3. The library search accuracy at various ranks, for various similarity measures for 10 subsets of replib which were used for cross-validation. For composite similarity measure: $a=1.0, b=$ $d=0.5$. Stereoisomers were treated as different compounds. 